This will not put urologists out of work; patients with poorly controlled disease will still tax their skills. Identifying and preventing tumour progression remains a problem. With a reduction in the time spent on unnecessary cystoscopies the management of superficial bladder cancer will be better for patients and more rewarding for urologists.

One final point. In private medicine a patient with a bladder tumour is considered a good investment who will provide a steady income for several years. Similarly, urological provider units will now gain much of their income from managing superficial bladder tumours. In future, purchasers of health care may well express an interest in how this is done.

DAVID KIRK Consultant urologist
1 Greene LF, Hanash KA, Farrow GM. Benign papilloma or papillary carcinoma of the bladder? f Urol 1973;110:205-7.

Cutler SJ, Heney NM, Freidell GH. Longitudinal study of patients with bladder cancer: factors associated with disease recurrence and progression. In: Bonney WW, Prout GR, eds. Bladder cancer. Baltimore: Williams and Wilkins, 1982:35-46. (AUA Monograph Vol 1).

3 Abel PD. Follow-up of patients with "superficial" transitional cell carcinoma of the bladder: the case for a change in policy. $B r f$ Urol (in press).

4 Gulliford MC, Burney PGJ, Petruckevitch A. Can efficiency of follow-up of superficial bladder cancer be increased? Ann R Coll Surg 1993;75:57-61.

5 Tolley DA, Hargreave TB, Smith PH, Williams JL, Grigor KM, Parmar MKB, et al. Effect of intravesical mitomycin $\mathrm{C}$ on recurrence of newly diagnosed superficial bladder cancer. $B M \mathcal{F}$ 1988;296:1759-61.

6 Fowler CG, Badenoch DF, Thakar DR. Practical experience with flexible cystoscopy in outpatients. Brf Urol 1984;56:618-21.

7 Abel PD. Prognostic indices for superficial bladder cancers. In: Waxman J, Williams G, eds. Urological oncology. London: Edward Arnold, 1992:154-66.

8 Heney NM, Nocks BN, Daly JJ, Prout GR Jr, Newall JB, Griffin PP, et al. Ta and T1 bladder cancer: location, recurrence and progression. Br f Urol 1982;52:152-7.

9 Parmar MKB, Freeman LS, Hargreave TB, Tolley DA. Prognostic factors for recurrence and followup policies in the treatment of superficial bladder cancer: report from the British Medical Research Council subgroup on superficial bladder cancer (Urological Cancer Working Party). f Urol 1989;142:284-8.

\title{
Screening for ovarian cancer
}

\author{
Still a long way to go
}

Because ovarian cancer is usually asymptomatic until widely disseminated it is commonly diagnosed at an advanced stage and is frequently lethal. Most early stage ovarian cancers are detected incidentally on routine pelvic examinations, and five year survival rates of over $90 \%$ can be achieved for localised disease. This fact, together with the minimal improvements achieved in the past 20 years in mortality with aggressive treatment of advanced disease, has awakened interest in the early detection of ovarian cancer by screening.

Ovarian cancer has a low incidence ( 15 per 100000$)$, which raises questions about the usefulness of screening in the general population (even though ovarian cancer is responsible for most deaths from genital tract malignancies in developed countries). To justify a screening programme, the disease needs to be reasonably common, and screening methods with a high degree of specificity and sensitivity need to be available. It also helps if there is an identifiable precursor of the invasive disease. Subsets of patients have been identified who are at a higher risk for developing ovarian cancer, and screening these patients may prove more cost effective.

Women with a family history of ovarian cancer are one such group. Studies suggest that women with one first degree relative with the disease have a 5\% risk of developing ovarian cancer'; with two affected close relatives they have about a $30 \%$ lifetime risk. ${ }^{2}$ Screening may therefore benefit these women but, even if screening was effective, its impact on the disease as a whole would be minimal, as familial cancers make up less than $3 \%$ of all ovarian cancers.

The only two screening tests for ovarian cancer that have been studied in any detail are pelvic ultrasonography and serum CA 125 concentrations. The two papers published in this week's journal use these techniques differently (p 1030, p 1025 ). The paper by Jacobs and colleagues deals with routine screening by measuring CA 125 concentrations in 22000 apparently healthy postmenopausal women. Those who had raised concentrations were then studied using real time transabdominal ultrasonography, and if this was abnormal, a laparotomy was performed. ${ }^{3}$ Of this large group who were screened, only 11 patients had raised CA 125 concentrations, an abnormal scan, and ovarian cancer at laparotomy. Of the 11 ovarian cancers, seven were stage III or IV. Seven patients with a normal serum CA 125 concentration subsequently developed ovarian cancer, two within 12 months of screening. Thus the protocol detected only four potentially curable cancers (the ultimate reason for screening), detected seven incurable tumours, and failed to detect another seven. At a conservative cost of $\$ 200$ per scan, the four potentially curable cancers were diagnosed at a cost exceeding $\$ 1 \mathrm{~m}$ a case.

The study by Bourne and colleagues used colour flow imaging with transvaginal ultrasonography targeted to patients with a family history of ovarian cancer. ${ }^{4}$ Of 1601 patients screened, $61(3.8 \%)$ had a positive result and had laparotomy or laparoscopy. In this group six ovarian cancers were diagnosed, five being stage I(a). Three of the five stage I patients had "borderline" tumours, which may have been diagnosed by subsequent clinical examination before spread occurred. In the intervals between screening, five ovarian or peritoneal cancers developed. The probability of detecting ovarian cancer using this technique was estimated to be 1 in 10 , improving to 1 in 6 if only complex persistent cysts were considered to be potentially malignant.

These two new studies suggest that we have a long way to go before effective screening for ovarian cancer is established. We have first to develop tumour markers or panels of markers with more specificity than CA 125 . It is estimated that CA 125 concentration is $>65 \mathrm{U} / \mathrm{ml}$ in only half of stage I and II ovarian cancers. ${ }^{5}$ Numerous benign conditions may cause false positive results and the positive predictive value of the test on its own is low. In a study of $\mathbf{5 5 0 0}$ patients screened with annual estimations of CA 125 concentration only six ovarian cancers were found out of 175 with raised concentrations and three cancers developed in the group with normal serum CA $125 .^{6}$

Screening using transvaginal ultrasonography seems to lower the rate of false positive scans when compared with abdominal ultrasonography ${ }^{5}$ but it is still not accurate enough as a screening technique. Because of the increased angioneogenesis of malignant tumours colour flow Doppler sonography has been used to distinguish between benign and malignant tumours, and a "morphology index,"-based on tumour volume, cyst wall structure, and structure of the septa-may increase the specificity of transvaginal ultrasonography as a screening method ${ }^{8}$. When this method was used to analyse scans on 3220 asymptomatic postmenopausal women, however, 44 patients had a laparotomy for a persisting abnormality, and only three had ovarian cancer, one of which was already stage III. ${ }^{9}$

At our present state of knowledge and available technology 
screening the general population seems neither useful nor cost effective. High risk patients should be encouraged to participate in large clinical trials to access the efficacy of newer screening techniques and, most importantly, to assess the effect of screening on mortality from the disease, as improvements in mortality have not yet been shown. In the future molecular genetic studies may be able to identify those patients at risk who may benefit from careful screening or prophylactic oophorectomy.

MAURICE J WEBB

Professor and chairman of gynecologic surgery

Mayo Clinic,

Rochester MN 55905,

USA

1 Lynch HT. Genetic Risk in Ovarian Cancer. Gynecologic Oncology 1992;46:1-3.
2 Ponder BAJ, Easton DF, Peto J. Risk of ovarian cancer associated with a family history. In: Sharp F, Mason WP, Looke RE, eds. Ovarian cancer-biological and therapeutic challenges. Londo Chapman and Hall, 1990:3-6.

3 Jacobs J, Prys Davies A, Bridges J, Stabile I, Fay T, Lower A, et al. Prevalence screening for ovarian cancer in postmenopausal women by CA 125 concentration and ultrasonography. BMJ 1993;306:1030-4.

4 Bourne TH, Campbell S, Reynolds KM, Whitehead MI, Hampson J, Roystor P, et al. Screening for early familial ovarian cancer with transvaginal ultrasonography and colour flow imaging. $B M \mathcal{Y}$ 1993;306:1025-9.

5 Zurawski VR, Knapp RC, Einhorn N, Kenemans P, Mortel R, Ohmi K, et al. An initial analysis of preoperative CA 125 levels in patients with early stage ovarian carcinoma. Gynecologic Oncology 1988;30:7-14.

6 Einhorn N, Sjovall K, Schoenfeld DA. Early detection of ovarian cancer using the CA 125 radioimmunoassay. American Society of Clinical Oncology 1990;9:A607.

7 Van Nagell JR, DePriest PD, Puls LE Donaldson ES, Gallion HH, Pavlik EJ, et al. Ovarian cance screening in asmpematic postmenopausl women by tansaginal ulrasound. Caner 1901;68: 458-62.

8 DePriest PD, Shenson D, Fried A, Hunter J, Andrews SJ, Gallion HH, et al. A Morphology Index based on sonographic findings in ovarian tumors. The 24th Annual Meeting of the Society of Gynecologic Oncologists, Program Abstract No 27, 1993.

9 DePriest PD, Gallion HH, Hunter JE, Andrews SJ, Parelick EJ, Van Nagell JR. Transvaginal sonography (TVS) as a screening method for ovarian cancer in asymptomatic postmenopausal women. The 24th Annual Meeting of the Society of Gynecologic Oncologists, Program Abstract No 21, 1993.

\section{After Concorde}

\section{Suggests no place for zidovudine as sole treatment for asymptomatic HIV positive patients}

The results of an important trial summarised earlier this month in a letter to the Lancet are discussed here before further analysis, peer review, and full publication because of their potential impact on patient care and the design of further antiretroviral studies. ${ }^{12}$ The Anglo-French Concorde study was initiated in 1988 and enrolled more than 1700 asymptomatic patients infected with HIV into a trial of immediate versus deferred treatment with zidovudine.

Patients randomised to deferred treatment received a placebo until the onset of clinical symptoms related to HIV infection (Centers for Disease Control group IV), ${ }^{3}$ when all were offered open label zidovudine. After a change to the protocol in 1989 patients were also eligible to receive zidovudine when the CD4 lymphocyte subset ( $T$ helper cell) count fell below $500 \times 10^{\circ} / 1$. No differences were seen between the two groups in development of clinical symptoms or survival over a mean follow up of three years. In the early treatment group the CD4 count had increased by a mean of $20 \times 10^{\circ} / 1$ cells three months after enrolment, but the count had fallen by $10 \times 10^{6} / 1$ cells among those in the deferred treatment group. This 30 cell difference was sustained throughout follow up.

These results do not challenge the value of zidovudine in prolonging the life of patients recovering from pneumocystis pneumonia, delaying the development of opportunistic infections in symptomatic patients with HIV infection, ${ }^{4}$ and improving the cognition of those with AIDS dementia complex. ${ }^{5}$

Given this background of clinical benefit among symptomatic patients with HIV infection, there are several reasons why use of zidovudine at an earlier stage of disease was and continues to be logical. Although viral load measured in peripheral blood mononuclear cells is low in the asymptomatic phase, active replication continues in lymph nodes. ${ }^{67}$ Effective early treatment may arrest or at least slow the progressive fall in CD4 lymphocyte count before the onset of severe, possibly irreversible, immunodepletion. Less virulent strains of HIV that predominate in earlier disease (for example, non-syncytia inducing strains) may be more susceptible to antiviral drugs than those variants more prevalent in advanced HIV disease (syncytia inducing strains). ${ }^{8}$ Reduced sensitivity of viral isolates to zidovudine in vitro develops more slowly in patients treated at an early stage of disease than in those treated later. ${ }^{9}$ Zidovudine is less toxic when given to asymptomatic patients than when given to those with advanced disease. ${ }^{1011}$ Indeed, little toxicity was observed in the Concorde study despite the relatively high dose of zidovudine $(1 \mathrm{~g} /$ day) used.

The first studies of zidovudine treatment in asymptomatic patients and those with early symptoms organised by the AIDS Clinical Trial Group in the United States both showed a delay in the progression to AIDS. ${ }^{1011}$ These studies were criticised because of limited follow up and the relatively small number of events, particularly deaths. The Concorde study with its long follow up and large number of clinical events (404) shows that this early promise of zidovudine has not been translated into improved survival. Its organisers should be congratulated on their ability to bring this trial to a successful conclusion despite sometimes fierce criticism.

What should we tell our patients? Decisions about treatment by both doctor and patient require more information than is currently available, but the summary of the results of the Concorde study casts considerable doubt on the wisdom of using zidovudine as sole long term treatment in those with asymptomatic disease. Early use of antiviral drugs remains logical, and studies of combination and sequential treatment in these patients, such as the present European-Australian study, Delta, need to be vigorously pursued. If further analysis shows that a significant proportion of patients received zidovudine before a clinical end point one interpretation of the Concorde study would be that short term antiretroviral treatment producing transient reduction in viral load and improvements in CD4 count may be as effective as more prolonged treatment. Further controlled studies could investigate this.

The Concorde study is particularly important because it shows that reliance on the CD4 count as a surrogate marker of drug efficacy is premature. The minor improvements in CD4 count seen with early zidovudine treatment were not translated into increased survival or delay in the development of clinical events. The reasons for this are unclear: the rise in CD4 count may simply represent recruitment of cells into the circulation, some of which may be dysfunctional, or the numerical increase observed may be insufficient to influence outcome. Whatever the reason for the poor correlation between CD 4 cell count and clinical outcome, the Concorde 